\title{
La reconstrucción de postguerra y la construcción de la paz ${ }^{1}$
}

\author{
Lic. Fco. Javier Ibisate
}

La expresión de reconstrucción de postguerra daría a entender que la guerra es algo ya pasado que queremos olvidar, mientras que la tarea de construir la paz nos recuerda que la guerra es siempre un presente posible. Por ello preferimos hacer de la construcción de la paz el objetivo principal de la reconstrucción de postguerra y que ello inspire las remodelaciones económicas y las reformas civiles que nos lleven hacia, "una democracia económica humana" (Ota Sik). Se parte de la siguiente premisa: la paz ni se firma, ni es una firma; la paz se constnuye poniendo fin a las causas que construyeron la guerra. $Y$ parece que bastante nos cuesta dar el primer paso, la firma de la paz.

Estamos terminando el siglo- $X X$, a lo largo del cual lo que más se ha desarrollado ha sido la guerra y la producción para la guerra: dos devastadoras guerras mundiales, una gravosa carrera armamentistica que ha dejado superendeudadas a las dos grandes superpotencias, y también por carambola a las minipotencias; de 1950 a nuestros dias se han librado unos 150 conflictos bélicos civiles, la mayoría en el escenario del tercer mundo. Por desgracia, el siglo- $X X$ nos ha habituado a hacer y a resolver los problemas por la guerra: queremos la paz, pero hacemos la guerra. Ahí está, como reciente botón de muestra, la insensata "tormenta del desierto", condenada también por el Papa en su última encíclica. Volvemos a reeditar el antiguo adagio romano: "si vis pacem,

1 Ponencia presentada en el Seminario: "La reconstrucción de postguerra: el desaflo económico y social", organizado por CENITEC, el dla 27 de agosto de 1991. 
para bellum": si quieres la paz, prepara la guerra. También nosotros hacernos el diálogo para detener la guerra y hacemos la guerra para detener el diálogo.

En orden a responder al presente tema, la reconstrucción de postguerra, hay que responder con sinceridad a una doble pregunta: ¿por qué surgió nuestro conflicto civil de oflce años? y ¿once años de guerra han sido la solución? - La preguntà'y las' respuestas son importantes por una sencilla razón; al estar, primero Dios, en visperas de firmar la paz permanecen intocadas y empeoradas las causales que dieron lugar a la guerra: la pobreza generalizada, las discrepancias en los géneros y modos de vida y el descuido e irrespeto de los fundamentales derechos civiles, tal como consta en la agenda repetida del diáloggo. Si no logramos un cierto acuerdo sobre el porqué se hizo la guerra, dificilmente llegaremos a un acuerdo sobre el cómo llevar a cabo la reconstrucción de postguerra, y más en concreto, qué objetivos económicos y sociales se van a preferenciar y qué mecanismos, políticas o modelos económicos se van a integrar.

Para entrar en el tema de la reconstrucción de postguerra y en cuanto la historia es la maestra de la vida me permito hacer un recuerdo personal de guerra y postguerra. A quienes nacimos en el año de 1930 , crisis mundial del capitalismo, no vamente seis anos de crisis y penuriä," sẹguidos de cuatro anos de guerra civil española, haciendo empalme inmediato con otros cinco años de segunda guerra mundial. Luego hubo que enfrentar la reconstrucción en un ambiente de penuria y sensible escasez, y al mismo tiempo habia que buscar la mútua colaboración nacional junto con un sacrificio generalizado; porque no puede haber reconstrucción sin mútua colaboración, ni mútua colaboración sin sacrificio compartido. En ese entonces se utilizaron ciertas medidas sociales y económicas con los que no parecen estar muy de acuerdo nuestras nuevas corrientes económicas. Pero merece la pena recordar la historia.

Primero, nuestro principal documento de identificación fué la "cartilla de racionamiento", igual para todos, aunque siempre hayan existido los privilegiados de siempre. Objetivo; que en medio de la penuria generalizada, a nadie faltara la oportunidad de satisfacer las necesidades minimas de la canasta familiar. Una reconstrucción de postguerra exige sacrificio compartido, horas y dias extras de trabajo (adios a los largos fines de semana con dos dias de ocio), que suplen la forzada escasez de capital técnico y capital financiero. Trabajo común, sacrificio común. En otras palabras, no se puede logar una equitativa reconstrucción de Dostguerra sin aceptar una primera etapa de económia de sencillez y 
sobriedad, que se centra en lo esencial para todos, y que por un tiempo relega al próximo futuro la producción de bienes y servicios más suntuarios o menos necesarios. En esta primera etapa no hay lugar para las "zonas rosas económicas". Ese era el obletivo de la cartilla de racionamiento, símbolo e instrumento de un sacrificio compartido.

Sin embargo, no estoy proponiendo ni recomendando que se nos imponga la cartilla de racionamiento, y ello por dos motivos bastante preocupantes. Primero, porque en nuestro medio ambiente la mera propuesta pareceria una aberración y una marcha atrás a quienes no están, - no estemos, dispuestos a este sacrificio comunitario en pro de una economía de mayor sobriedad en el género y estilos de vida, postergando lo suntuario y socialmente irritante. Creo que la Asamblea Legislativa no aprobarla tal decreto ni por la mitad más uno. Segundo, y es más preocupante, si se aprobara el decreto y se impusiera la cartilla de racionamiento, al modo del carnet electoral, no tendría el efecto deseado. En nuestra situación, la mayorla de la población no podría utilizar la cartilla de racionamiento porque siguen presentes las causales de la guerra: el escaso poder de compra derivado de la pobreza y desempleo generalizados. Entre paréntesis, esto significa que falta la base fundamental para llegar a una economía de mercado a través de una economía de mercado cuando la mayor parte de la población se presenta como una fuerza de demanda desarmada. Precisamente, este deberla ser el objetivo principal del plan de reconstrucción de postguerra: lograr to que buscaba la cartilla de racionamiento, que todos puedan participar de los frutos de la reconstrucción, en la satisfacción de las necesidades humanas, económicas y civiles. Si con la reconstrucción no se logra este objetivo, aunque se firme la paz estaremos construyendo la guerra.

Un segundo recuerdo de aquellos años de postguerra fué el renacimiento y fuerte impulso del análisis y enfoques macroeconómicos, luego de trece crisis en las economias de mercado, con una mayor participación orientadora y activa del Estado, por medio de los Planes de Desarrollo, -apoyados normalmente en ese instrumento de análisis y planeación económica que son las matrices intersectoriales $W$. Leontief. Se buscaba con ello que, así como a nivel de empresa la planeación es la etapa básica de la organización, ejecución y control de la producción, en forma semejante los planes de desarrollo nacional orientaran y activaran un crecimiento armónico e integrado de todas las ramas- sectores productivos, en orden a fortalecer la interdependencia de los mismos y lograr una estructura económica más consolidada.

Estas teorias y prácticas económicas las hemos integrado con un cierto retraso y baja intensidad en El Salvador. Nuestros primeros Pla- 
nes de Desarrollo datan de 1960, aunque han, sífo mnás bien simples diagnósticós que verdaderos planes de desarrollo, poràùe la economla real avanizada por la acera de enfrente haciendo oldos sordos a los objetivos planeados. También, no ha sido sino hasta 1986 que el Banco Central pudo elaborar la matriz insumo-producto 1978, y actualmente trabaja en la edición de matrices para anos más recientes, 1984, 1985, 1986... La razón de esta cita es que el aporte parcial de la presente ponencia al tema de la reconstrucción de postguerra se basa en las orientaciones técnicas que nuestra matriz intersectorial ofrece tanto al Plan de Desarrollo como a un mecanismo de mercado que quiera seguir los lineamientos de una "economla social CON mercado".: (ECA, 1989; pp. 311-336)

A primera vista, por una interpretación parcial y parcializada del dernumbe de las economías del Este, se les llame socialismo reales o socialismos irreales, se tiende a desacreditar o rélegar a un lugar secundario las funciones del Estado, y con ello el papel hnás activo de los planes de desarrollo. En segundo lugar, el eje principal de la economla tiende a pivotear en un mercado, enfatizando el desarrollo hacia-fuera, en orden a hacer más agresiva, más competitiva a la empresa naclonal, y llevarla hacia escalas de mayor producción y menores costos marginales ante un mercado externo ampliado. Por la ley del péndulo, que también juega en economia, se tiende a contraponer el modelo de desarrollo hacia-fuera y el modelo de desarrollo hacia-dentro, a contraponer mercado externo y mercado interno nacional.

Pero si la causal fundamental de nuestra guerra fué, es y seguirá siendo el desarrollo de nuestro mercado interno, y no sólo en lo referente a nuestra canasta básica sino al resto de los derechos civiles, hay que hacer los mejores esfuerzos por integrar el desarrollo endogeno, hacia-dentro, con el imprescindible desarrollo hacia-fuera, sin el cual imposible subsistir. Poniendo la tesis en palabras más concretas, un desarrollo endógeno (hacia-dentro) que busca la satisfacción de las necesidades básicas ¿colaboraria a generar una reactivación económica?- Esto es lo que se pretende afirmar y mostrar a partir de un sencillo ejercicio práctico, que iniciamos en el Departamento de Economla, luego de la aparición de la matriz insumo-producto 1978. Este ejercicio práctico no es un modelo completo, sino un aporte parcial a integrarse en un modelo ampliado macroeconómico que busque integrar el desarrollo hacia-dentro con el desarrollo hacia fuera: conjugar y no contraponer los enfoques.

La hipótesis económica, tal como la presenta nuestro Instituto de investigaciones económicas (IIES) (Realidad Económica-Social, 1991, 
NN 21), es que un modelo económico que busca la erradicación de la pobreza obedece a dos principios: a) un crecimiento económico fuerte y sostenido, acompañado de niveles elevados de empleo, y b) una distribución más equitativa del ingreso y de los frutos del crecimiento. Citando literalmente se nos dice: "Esto es as' porque el desarrollo económico de un pais no puede reducirse al sólo aumento de la producción de bienes y servicios, ni tampoco a una distribución más equitativa de un ingreso insuficiente. Dicho de otro modo, no todo proceso de crecimiento significa desarrollo, pero todo proceso de desarrollo requiere de crecimiento económico. También es evidente que entre los objetivos de un crecimiento económico fuerte y una distribución más equitativa de los frutos del mismo pueden presentarse conflictos. Las estrategias de desarrollo o los proyectos de nación se diferencian fundamentalmente por la forma en que se resuelven o plantean resolver esos conflictos".

El problema concreto es si, en la elaboración de tales proyectos y estrategias, simplemente nos ponemos de acuerdo o desacuerdo entre economistas y altos funcionarios, o más bien si somos capaces de escuchar y asumir las expectativas de las mayorias, quienes al fin y al cabo o nos van a aceptar o nos van a rechazar el modelo. Frente a estas posibles expectativas la hipótesis de esta ponencia es que un modelo económico que preste atención al desarrollo endógeno y trate de responder a las necesidades básicas es un proyecto y una estrategia de reactivación económica. En otras palabras, el desarrollo endógeno es un complemento necesario para un desarrollo con crecimiento económico y distribución más equitativa.

La parte inicial de este trabajo quedó sintetizada en nuestro Boletín de CC. Económicas y Sociales, julio-agosto de 1987, como resumen de un trabajo de investigación. ${ }^{2}$ El análisis ecométrico utilizó como instrumento técnico la "matriz insumo-producto 1978 y el juego secuencial del efecto-multiplicador del gasto. A cada nivel de avance en el ejercicio práctico se hacen aplicaciones derivadas de las virtualidades o usos múltiples propios de estas matrices intersectoriales. Un objetivo práctico y concreto es el de ayudamos a consolidar nuestras estructura productiva y con ello fortalecer el desarrollo hacia-fuera reformulando el desarrollo hacia-dentro. Cuando el BCR edite las nuevas matrices intersectoriales será preciso hacer una actualización del presente ejercicio.

2 Orellana Alba M" y Alfaro José N.: "Necesidades básicas y reactivación de la Economla". Tesis de Grado. UCA. San Salvador, julio de 1987. Resumen. Boletin de CC. Económicas y Sociales; julio-agosto 1987. 


\section{Necesidades błsicas y reactlvación de la economla}

\section{Formulaclón del modelo}

El ejercicio práctico arranca con la implementación de una politica de distribùción o redistribución de ingresos, via la generación de empleo por un monto de $\$ 100$ millones. No se trata en èste supuesto de una transferencia de ingresos de los que tienen más a los quétienen menos, sino de una distribución o encauzamiento de ingresos (préstamos, donaciones, ayudas...) hacia un grupo de inactivos de mayor propensión a consumir. Es en este sentido que los cálculos a derivar indicarán también los efectos positivos de una política necesaria de redistribución de ingresos.

La formulación del modelo permite calcular los efectos de un aumento en la demanda final dirigida a las ramas productoras de bienes y servicios sobre la producción de las otras ramas de actividad económica que conforman la estructura productiva de EI Salvador. La utilidad de la matriz insumo-producto radica en que ella ofrece una cuantificación de las relaciones intersectoriales, en valores absolutos y en coeficientes técnicos, y permite examinar las repercusiones que sobre cada sector tendrá una modificación en la demanda final; asl como la necesidad de aumentar la producción de los diversos tipos de bienes que la integran a consecuencia de un incremento en el ingreso per-capita. El uso de la matriz requiere de ciertos presupuestos, que luego se explican. Pese a ciertas restricciones que pueden generar estos supuestos, el modelo pone de manifiesto los puntos más importantes de la dinámica económica. Una política redistributiva, al aumentar la demanda final de bienes y servicios básicos, que satisfacen las necesidades mínimas de las mayorías populares, tendrá una serie de efectos directos e indirectos sobre las ramas que conforman el aparato productivo de la economla salvadorefa. Estos efectos son calculados utilizando la matriz inversa o matriz de coeficientes técnicos directos e indirectos.

\section{Supuestos generales que prevalecerán constantes}

- Se utiliza la matriz insumo-producto 1978 , editada por el BCR, tomando como relativamente constantes los coeficientes técnicos de los insumos combinados por cada rama, los coeficientes técnicos del valoragregado y los coeficientes técnicos de la matriz inversa. Esto implica que no ha habido transformaciones notables en la tecnologia de producción sectorial. Una vez publicadas las matrices insumo-producto del BCR para años más recientes habria que verificar estos supuestos.

- Se supone (supuesto importante) que la oferta responderá ade- 
cuadamente a los niveles requeridos por el aumento de la demanda final de bienes y servicios. Este supuesto es importante, no porque la oferta responde a tales demandas, sino porque sirve de pauta a las políticas complementarias tendientes a estimular esta producción. Cómo se reactivaría la economia si dichos sectores responden a las demadas básicas.

\section{Supuestos especiflcos}

Partimos del supuesto de que este proyecto de inversión y gasto inicial se prolonga por varios anos, hasta que el juego del efecto multiplicador se desvanezca; a cada ano le damos el subindice " $z$ " I, 2, 3...

El ejercicio se inicia con un gastos-inversiones de $\$ 100$ millones,que genera ingresos equivalentes en grupos inactivos de mayor propensión a consumir. En fase de reconstrucción de postguerra se hacen urgentes múltiples inversiones en trabajos públicos, en forma centralizada o descentralizada, dentro de las áreas senaladas en nuestro Plan de Desarrollo Social; inversiones directamente ligadas a la satisfacción de las necesidades básicas y a una reestructuración geográfica de la población y nuevos polos de desarrollo. Seria de desear que quienes nos han prestado una "ayuda militar" nos colaboraran con una sensible y necesaria ayuda económica para que la guerra no vuelva a rebrotar.

La fuerza de trabajo, aqui contemplada, ha estado o está totalmente inactiva (supuesto bien realista), y por lo tanto todos.sus ingresos pasan a consumo. Siendo su propensión marginal a consumir igual a I (PMC1), tenemos que los ingresos de los trabajadores son igual que el consumo de los trabajadores ( $Y L=C L$ ), siendo cero el ahorro $(A L=O)$

Las ramas de actividad involucradas en este primer periodo (representadas con el subindice " $n$ "), de acuerdo a la nomenklatura de la matriz 1978 son los siguientes 17 sectores productivos: 3, 5, 7, 11, 14, $15,16,17,20,25,26,32,33,37,41$ y 44 ; ellos responden a las demandas integrantes de la canasta básica.

\section{Aspectos metodologlcos}

Para determinar la distribución e irıcremento de la demanda final dirigida a cada una de las 17 ramas afectadas se utiliza el gasto normativo mensual de la canasta básica y la estructura promedio de una serie histórica para los sectores populares, de acuerdo al estudio sobre "La determinación del consumo mínimo en El Salvador". De acuerdo a la estructura porcentual de distribución de la demanda, los $\$ 100$ millones pagados a los nuevos trabajadores se distribuirian a su vez, entre los sectores básicos, en la forma siguiente: 
Aumento de la demanda final de bienes y servicias básicos

\begin{tabular}{|c|c|c|}
\hline \multicolumn{2}{|c|}{ Ramas } & Aumento demanda final en colones \\
\hline 3 & 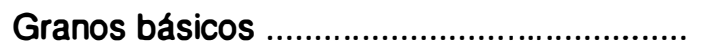 & $6,200.000$ \\
\hline 5 & 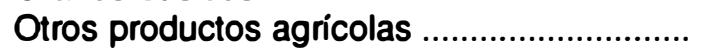 & $10,900.000$ \\
\hline 7 & 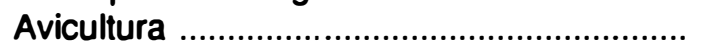 & $5,500.000$ \\
\hline 11 & Carne & $1,900.000$ \\
\hline 12 & 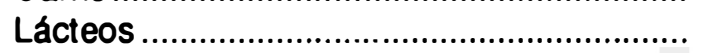 & $6,600.000$ \\
\hline 14 & 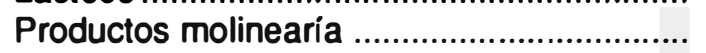 & $5,600.000$ \\
\hline 15 & 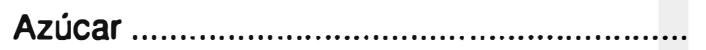 & $1,100.000$ \\
\hline 16 & 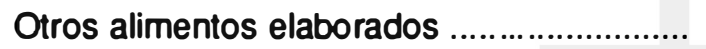 & $6,200.000$ \\
\hline 17 & 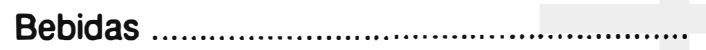 & $1,500.000$ \\
\hline 20 & Vestuario & $4,000.000$ \\
\hline 25 & Químicos ......................... & $2,900.000$ \\
\hline 26 & 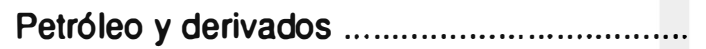 & $3,600.000$ \\
\hline 32 & 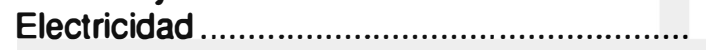 & $1,200.000$ \\
\hline 33 & Agua & $1,700.000$ \\
\hline 37 & 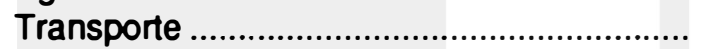 & $5,600.000$ \\
\hline 41 & Alquiler de vivienda & $30,900.000$ \\
\hline 44 & Servicios de Gobierno (salud, educac.) ........ & $4,600.000$ \\
\hline & ........ 1 & 100.000 .000 \\
\hline
\end{tabular}

La demanda total a cada rama productiva se ha derivado de la distribución porcentual normativa de la canasta básica. A la cantidad de demanda final, en valores absolutos, dirigida a cada sector le asignamos la sigla "Q", utilizada en posteriores fórmulas del ejercicio.

\section{Cálculo de los efectos derlvados}

Partiendo de este gasto inicial nos interesa especificamente calcular los siguientes efectos sobre el conjunto de la economia:

a) El efecto producción

b) El efecto empleo

c) El efecto: Ingreso de trabajadores; c. 2 de Empresarios.

d) El efecto en el sector externo: Importaciones.

A) El efecto producción. Mide el efecto de esta demanda final sobre las 44 ramas productivas como abastecedoras directas 0 indirectas de las 17 ramas primeramente afectadas. Para ello se utiliza la matriz de coeficientes técnicos inversos. 
(Formulación. $(P n) z=(A i n)^{-1} \cdot Q$. Donde $(P n) z$ es la producción total del sector " $n$ " en el periodo " $z$ ", debido al aumento de la DFn en el perlodo " $z$ ". (Ain)-1, es la columna de la matriz inversa de la rama " $n$ " $(1,2,3,4, \ldots)$. "Q" es demanda final del sector " $n$ ".)

B) El efecto empleo. Mide el empleo requerido en las 44 ramas productivas para responder al correspondiente aumento de producción.

(Formulación. $(E n) z=(P n) z$. $(T n)$. Donde $(E n) z$ es la cantidad de empleo requerido por la rama " $n$ " debido al aumento de la producción (Pn)z. (Tn) es el coeficiente técnico del factor trabajo, siendo (Tn) la cantidad de trabajo necesaria por unidad de producción, medido en horas-hombre utilizadas: $(T n)=H n / P n$.

C) El efecto ingresos. Mide el efecto que sobre los ingresos de los trabajadores y de los empresarios tiene el incremento de empleo. En cada caso se utilizan los coeficientes técnicos del valor agregado para trabajadores y el excedente de explotación para los empresarios.

(Formulación. C. 1 Trabajadores: (YLn)z $=$ (En/8 hrs). Jn. El empleo se calcula en dias-hombre trabajo y se multiplica por el salario correspondiente para calcular los ingresos.

C. 2 Empresarios. (YEn)z = (Pn)z. (Gn). Los ingresos de los empresarios se miden por el aumento de sus excedentes, debido al aumento de la producción y del empleo, siendo (Gn)z el coeficiente técnico del excedente de explotación.)

D) El efecto en el sector externo: importaciones. Mide el aumento de la demanda de importaciones requerido por las 44 ramas para responder al incremento de producción.

(Formulación. $(M n) z=(P n) z$. Lin). Donde $(M n)$ es el incremento de las importaciones de cada rama productiva, debido al aumento de la producción, y (Lin) es el coeficiente técnico de insumos importados para cada rama " $n$ " de la economia.)

En la aplicación de las fórmulas se hicieron algunos supuestos que no influyen sensiblemente en los resultados globales. Por ejemplo que la distribución de "suelos-salarios" del valor agregado es de un $40 \%$ para técnicos y empleados administrativos, $60 \%$ para el resto de trabajadores manuales. Los primeros consumen de acuerdo a la PMC promedio nacional, los segundos tienen una $P M C=1$. Igualmente se hicieron cálculos sectoriales de los coeficientes técnicos del factor-trabajo" por unidad producida, a partir de las referencias disponibles. Se buscaba con ello la mayor aproximación posible. 
6. Metodölogla para el cálculo del efecto-multipllcador do la polittca en la economla

Una vez que los $\$ 100$ millones han sido dedicados, en un primer perlodo, a gastos de consumo de bienes y servicios básicos, cuya producción ha generado a su vez incrementos de producción en el conjunto de sectores económicos y los correspondientes ingresos en los factores de producción, el efecto multiplicador empieza en un segundo perlodo $(z=2)$ y continúa actuando en forma decreciente durante varios perlodos, que abàrca la política, afectando a las variables antes mencionadas: producción, empleo, ingresos e importaciones.

El valor del efecto-multiplicador es directamente proporcional a la propensión marginal a consumir $(k=1 / 1-c)$, tomando en cuenta otras fugas que aminoran su efecto: los impuestos, las importaciones, máxime en una economia tan abierta. En nuestro ejercicio se observa que este juego del multiplicador se prolonga durante otros cinco anos.

\section{Resultados y análisls de los resultados}

Resumiendo los efectos totales sobre cada una de las 44 ramas productivas $^{3}$ se presentan los resultados anuales para las cinco variables calculadas.

\begin{tabular}{|c|c|c|c|c|c|}
\hline Perlodo & p-producción & $\begin{array}{l}\text { Empleo (D/h) } \\
\text { (Dias-hombre) }\end{array}$ & $\begin{array}{l}\text { Ingreso } \\
\text { trabajo }\end{array}$ & $\begin{array}{l}\text { Ingreso II } \\
\text { Empresa }\end{array}$ & nportación \\
\hline & & & 15,02 & & \\
\hline & & & & & \\
\hline & & & & 1.803 & 5,593 \\
\hline & $16,133.768$ & & 1,66 & 9.770 & \\
\hline & & & & 79.770 & $1,178.9$ \\
\hline & 20695 & 66.707 & 350.693 & $1,197.089$ & 541.282 \\
\hline
\end{tabular}

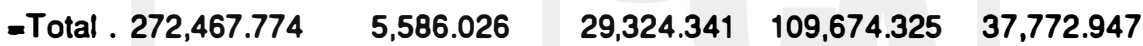

Los resultados vienen expresados en millones, excepto el empleo en dlashombre-trabajo.

El objetivo del presente ejercicio era investigar cuál serla la capacidad de reactivación económica de un modelo que respondiera a la satisfacción de las necesidades básicas, y por lo tanto si es factible conjugar las políticas de redistribución de ingresos, satisfacción social y

3 El cuadro detallado para las 44 ramas en Boletín de CC. Económicas y Sociales; julio-agosto, 1987; p. 239. 
reactivación de la economia. Los resultados del ejercicio vienen a confirmar las hipótesis iniciales y puede integrarse en un modelo más ampliado macroeconómico. En este caso tendriamos un efecto multiplicador de 2'725; proporción elevada en que se incrementarla la producción total, tomando en cuenta nuestra estructura interna trunca y dependiente, aparte de los efectos sobre el empleo, los ingresos y relativamente menores importaciones.

Hay dos explicaciones que pueden servir de orientación tanto a nuestro Plan-Desarrollo como a nuestro mercado. En primer lugar, se parte del supuesto de que el gasto 0 inversión inicial crea directamente ingresos en sectores desempleados, quienes tienen una elevada propensión marginal a consumir; transforman inmediatamente sus ingresos en demanda. Este es un dato a favor de tantas inversiones que actualmente se programan en sectores y áreas marginadas, a condición de que los nuevos ingresos no se dediquen a remunerar demasiados funcionarios y administrativos.

En segundo lugar, nuestra matriz 1978 muestra que el conjunto de sectores económicos que responden a la satisfacción de las necesidades básicas contienen una elevada capacidad de reactivación económica por sus encadenamientos "río-arriba" (como demandantes de insumos) y "río-abajo" (como oferentes de insumos a otros sectores). Ellos pueden colaborar a montar una estructura económica más integrada, apoyando tanto el desarrollo hacia-dentro como el desarrollo hacia-fuera, y relativamente con menor dependencia externa de importaciones. Si recogemos en un cuadro4 los 15 sectores más importantes por el ValorBruto-Producción, por el Valor-Agregado, y de manera especial los sectores claves como demandantes y como oferentes de insumos, se observa que estos sectores sociales (que responden a las necesidades básicas de la población) ocupan puestos primordiales, conjugando el doble aspecto de ser sociales y ser activantes de la economia.

En un proceso de "reconstrucción económica y social" se debe prestar atención al hecho de que el desarrollo endógeno no es contraproducente ni opuesto al desarrollo hacia-fuera. No juega aqui la "ley del péndulo" que nos lleve a uno de los dos extremos; por donde más veces pasa el péndulo es por el centro. De manera especial estas caracteristicas debieran estar presentes en el proceso de reestructuración bancaria, que en su estado de quebranto financiero tenderá a guiarse por el criterio"productividad rentable" de corto plazo. Pero conviene combinarlo

4 Ibisate Fco. J. "La ley de privatización bancaria: ¿de la privatización a la privatización?. Realidad Económico-Social. Julio-agosto 1990; p. 348. 
con el criterio de "rentabilidad reproductiva;" dando un tratamiento especial.a los sectores con capacidad de dinamización económica y social.

Existen otras razones que avalan la importancia de estos sectores básicos afectados por el gasto inicial: granos básicos, otros productos agricolas, ganaderia, avicultura, carne, lácteos, productos de molineriapanaderia, azúcar, otros alimentos elaborados, bebidas, textiles, prendas de vestir, quimicos, petróleo, eléctricidad, construcción, transportealmacenamiento, bienes inmuebles y servicios, servicios comunales sociales y personales, alquileres-vivienda y servicios de gobierno. La mayoria de estas ramas - exceptuando químicos, petróleo, transporte...utilizan más bien materias primas nacionales; en otras palabras, utilizan relativamente menos insumos importados que otros sectores económicos, de acuerdo a los coeficientes técnicos de la matriz 1978. Dado que una estructura de producción es relativamente estable se puede concluir, con suficiente realismo, que la satisfacción de las necesidades básicas de las mayorias dinamiza la producción de las ramas que emplean relativamente menos insumos importados o principalmente recursos nacionales; por lo tanto contribuyen a aumentar el empleo y los ingresos de los sectores populares.

Uno de los usos que ha tenido la matriz intersectorial en otros paises es que ha servido como agenda de diálogo entre las dependencias ministeriales afines al proceso económico y los representantes empresariales-laborales de las ramas-sectoriales productivos. Dado que los sectores internos son interdependientes e interrelacionado, la matriz ayuda a conjugar los planes de producción respectivos, de manera que no se generen ni cuellos de botella ni invendidos, y se pueda proyectar las necesiddes de importaciones y exportaciones para lograr un crecimiento armónico. En ese diálogo se puede apreciar la vocación y dotación hacia-dentro y hacia-fuera de cada sector. Disponemos, en principio, del instrumento orientador si nuestro mercado quisiera ser a la vez eficiente y social. En concreto, nuestra matriz nos muestra que este conjunto de sectores nombrados acumulan muchos puntos en los cuatro efectos de producción, empleo, ingresos laborales y empresariales. (Boletin; idem; pp. 242-243)

\section{Matrlces de bloques-Industrlales: famllias de Industrlas}

Una de las finalidades de las matrices, en la mente de $W$. Leontief, era ayudar a construir bloques industriales, es decir, acercar geográficamente familias de industrias afines por el proceso de encadenamiento rlo-arriba y rio-abajo. Aplicado a nuestro caso esto tiene una doble finalidad. Sabemos que nuestros paises en desarrollo presentan una es- 
tructura de producción "trunca" porque nos han concentrado en la exportación de productos primarios, en su estadio primitivo, obligados a importar las mayores cantidades de insumos industriales. Esto no se corrige de la noche a la mañana. Por otra parte, presentándose en el campo el problema del desempleo en forma más severa, porque las exportaciones primarias crean desempleo estructural, la mano económicamente activa tiende a aglomerarse en la capital, sumado ahora el efecto del conflicto bélico. Por lo tanto, enfrentamos ahora el doble reto de buscar encadenamientos rio-arriba y rio-abajo, que favorezcan una agroindustria más bien localizada en el campo.

Los aportes de nuestra matriz insumo-producto nos orienta para hacer análisis más detallados de estos encadenamientos posibles, partiendo los unos del sector primario, otros del secundario y otros del terciario. A modo de ejemplo y utilizando los sectores que responden a las necesidades básicas, se pueden ir formando cadenas entre los siguientes sectores:

a) Sector agrario: otros productos agrícolas, granos básicos, ganaderia, avicultura...

b) Sector industrial; alimentos básicos: otros productos alimentarios elaborados, productos molineria-panaderia, lácteos, carnes y derivados, bebidas...

-Otros productos industriales: quimicos de base y elaborados, textiles, petróleo y derivados, prendas de vestir...

c) Sector servicios: alquiler de vivienda, transporte-alacenamiento, servicios comunales sociales-personales...

El ejercicio arrancaría del sector agricola, el cual se constituye como fundamental y estratégico para la satisfacción del consumo básico de alimentos, sea como productor directo, sea como suministrador de tales insumos al sector industrial. Esta cadena mereceria especial importancia.' De momento, la atención y la producción de estos productos alimentarios básicos es relativamente marginal.' Esto se debió anteriormente al carácter monoexportador de productos tradicionales. Ahora, el énfasis en la diversificación agraria para fomentar exportaciones no-tra-

5 Boletin, ibidem; pp. 244...

6. Donis Jorge A.: "Estructura y articulación agroindustrial en el Salvador". Tesis de Grado. UCA. Octubre de 1988.

7. Pleitez Rafael, et alii: "Implicación de los P.A.E. sobre los pequeños productores de granos básicos en El Salvador. Tesis de Grado. UCA. Septiembre de 1991 
dicionaies tiene como ventaja macroeconómica el que puede aliviar el déficit de la balanza comercial; tiene el posible atractivo de una mayor rentabilidad monetaria (si bien los más beneficiados parecen ser los mercaderes" exportadores, no tanto los directamente productores), y 'tiene como ventaja el inducir a nuevos tipos de cultivos. Al mismo tiempo, presenta la desventaja de debilitar el encadenamiento de sectores internos, agrario-industrial, quien a su vez necesitará incrementar la demanda de mayores insumos pagados a precio de divisa-externa. Ya en la matriz 1978 se aprecia que la mayoría de las ramas de la industria alimentaria dependia sensiblemente de insumos importados y de alimentos terminados que no están al alcance de las clases populares.

Más que difícil es imposible pretender una autarquia o autoabastecimiento de suministros básicos agrarios, en un país de tan reducida superficie y suelo tan deteriorado. Pero la necesidad de lograr una mayor seguridad alimentaria y de fortalecer la cadena interna agro-industria recomienda buscar un equilibrio entre el desarrollo hacia-dentro y el desarrollo hacia-fuera. Una reciente investigación sobre "La realidad agraria en El Salvador'" muestra con algunos ejemplos las ventajas económico-sociales de proceder a transformaciones internas de algunos productos que hoy por hoy exportamos en su estadio primitivo, adquiriéndoles posteriormente a precio de divisa externa con la transformación que le dieron en el exterior. Este encadenamiento interno mejoraria la cualidad de nuestras exportaciones tradicionales y no tradicionales, retroalimentándose ambos enfoques del desarrollo.

Otro sector básico importante es la "construcción de vivienda" accesible a la capacidad adquisitiva de los sectores populares, dado que esta rama satisface el $20 \%$ de la demanda final de bienes básicos. La rama "alquiler de vivienda" aparece, entre los sectores básicos, ocupado el primer lugar por su efecto producción y de generación de ingresos-empresa, junto con el tercer lugar como generación de empleo y de ingresos laborales. En cuanto sector construcción es un sector dinamizante de la economía como gran demandante de insumos; por ello podría ser uno de los sectores de donde arrancarias las inversiones de nuestro ejemplo de $₫ 100$ millones. Pero como alquiler de vivienda encuentra el obstáculo del costo de adquisición, elevados alquileres (más con la unificación del $20 \%$ del interés) y del financiamiento a largo plazo, dificultado con la reestructuración de las sociedad de ahorro y

8. Montoya Aquiles: "La realidad agraria de El Salvador". ECA. junio 1991; pp. 547-548... 
préstamo. ${ }^{9} \mathrm{He}$ aqui un caso conflictivo donde las nuevas medidas crediticias y las fuerzas del mercado pueden frenar el activismo de un sector eminentemente social. El tema de la vivienda requiere de elucubraciones especiales.

Otro $20 \%$ de la demanda final de bienes básicos se destina a sectores que es necesario estimular: la produccion de quimicos, textiles, prendas de vestir y petroleo. La rama de quimicos es importante, no sólo por satisfacer necesidades básicas (medicinas, detergentes...), sino también porque suministra insumos a casi la mitad de los sectores económicos; igual comentario merece el petróleo, con la salvedad de que ambos sectores requieren a su vez de mayor cantidad de insumos importados. Aqui se impone un recuerdo importante. Otro de los aportes de la matriz intersectorial es que nos descubre y describe cuantitativamente cuáles son las "importaciones-claves" para la economia; es decir, aquellas que siven insumos a mayor número de ramas productivas 0 son imprescindibles para la demanda final. Por la tanto, en un pais de escasas divisas, habria que destinar las divisas a estas importaciones claves, relegando toda importación de bienes suntuarios o no dinamizantes de nuestra economia. Una vez más, se presenta un conflicto entre la libre elección o libertinaje de las importaciones y la mayor reactivación económica junto con la satisfacción de las necesidades básicas. Esta elección es parte del sacrificio generalizado, sin el cual imposible la reconstrucción.

En la satisfacción de las necesidades básicas un sector que absorbe el $8 \%$ de la demanda final es transporte-almacenamiento. Es claro que ambos sectores crecen con el crecimiento general de la economia. Al igual que en el caso anterior, también requieren de insumos importados. Se trata de otra variedad de importaciones claves para el desarrollo económico, que a partir del sector terciario puede iniciar una cadena "rio-arriba" hacia el secundario industrial.

\section{La distrlbuclón de Ingresos laborales-empresarlales}

Esta distribución merece lugar aparte. En el cálculo de los ingresos utilizamos coeficientes técnicos del Valor-Agregado en sueldos-salariosprestaciones y del excedente de explotación. El resultado es que los ingresos de los empresarios más que triplican los ingresos de los trabajadores, aunque estos - a nivel nacional- eran 54 veces más en número. Esta distribución sive a probar dos cosas. Primero, que una

9. Fontg Marta G. et-alii: "Efectos de la aplicación de las medidas de liberalización financiera sobre el sistema de ahorro y préstamo". Tesis de Grado. UCA. Abril 1991. 
oconomía dedicada a satisfacer las necesidades básicas de la población genera más que suficientes ingresos a la clase empresarial; es decir, se trata de una producción social y rentable. Pero al mismo tiempo esto sive a probar la pasada y actual distribución desigual de los ingresos, si se mantienen los mismo coeficientes técnicos o si las actuales medidas económicas tienden a ello. Tal estructura de distribución de ingresos dificulta seriamente el juego de una política redistributiva, porque derivarla la demanda final hacia bienes y servicios no básicos, como los aqul contemplados. Esta desigual distribución de ingresos es una de las causas del escaso crecimiento interno de nuestra economia. Por lo tanto una adecuada política fiscal, sin desincentivar la inversión deberla ir mitigando esta desigual estructura distributiva.

Por anadidura, al ir examinando los sectores que responden a las necesiddes básicas de la población, de acuerdo a nuestra matriz intersectorial, hallamos que también en muchos de estos sectores sociales se da la concentración mono y oligopólica de productores. Ello explica que, incluso estos sectores sociales, no quieran responder adecuadamente a lo más básico; bajo la misma etiqueta producen bienes de una canasta familiar no al alcance de las clases populares. Por ello en nuestro medio histórico, la privatización ha venido sobrecargada de concentración. Parecería que no tenemos la base para hacer la reconstrucción general dentro de un marco de sacrificio generalizado.

\section{Consideración final}

Nuestro actual Plan de Desarrollo se divide en dos partes, que aparentemente combinarlan el crecimiefito con una distribución más equitativa. Pero no queda nada claro el ensamblaje de los objetivos.

a) El desarrollo, que se llama económico, confiado eminentemente a las leyes, es decir a las fuerzas de oferta-demanda del mercado.

b) La segunda parte, calificada como desarrollo social, se confla, no exclusiva pero si preferencialmente al subimperio del Estado, conjugando la administración centralizada y la descentralizada. Este subimperio abarca los rubros de la educación, salud-nutrición, vivienda, seguridadempleo, familia y el deterioro ecológico...

El peligro es que estas dos partes componentes se desintegren entre si y dentro de si mismas. En el compartimento llamado mercado, si privan los criterios de eficiencia y rentabilidad contable, no se responderá espontáneamente a las demandas de la canasta básica familiar porque carecen de poder de compra. EI PTB y el IVAE pueden seguir creciendo sin que por ello, al interior, aumente la producción-satisfacción 
de tales necesidades básicas. En el compartimento del desarrollo social el peligro está en cómo se entienda el principio de SOLIDARIDAD. Dadas las estrecheces financieras derivadas de la guerra, la "solidaridad" puede reducirse a "subsidiaridad fiscal" o donaciones externas. La expresión "solidaridad" de postguerra debe entenderse de parte del Plan y de parte del Mercado como SER SOLIDARIOS de las demandas básicas económicas y civiles, lo cual cambiaria toda la tónica del presente modelo.

Este trabajo pretende recordar que esos sectores, llamados sociales, son también económicos de pleno derecho porque responden a las necesidades de la mayoría poblacional y porque contienen una elevada capacidad de reactivación económica. Si la inversión inicial de nuestro ejercicio se ubicara en esas áreas mencionadas del desarrollo social (amén de tantas necesidades de infraestructura secundaria), el sector social podria convertirse en el catalizador del sector mercado e integrar lo que ahora parece permanecer desintegrado.

Finalmente, hay una razón de ética y equidad económica. Si en anos pasados y más ahora el modelo económico de mercado se sostiene en buena medida gracias a los $\$ 750$ millones de remesas de emigrantes y por lo tanto son los "pobres-dólares" quienes apoyan al modelo, es justo y equitativo que el modelo se acuerde de los pobres desde ahora. 